\title{
Fatal portal thrombosis after laparoscopic Nissen fundoplication
}

\author{
R. A. García Díaz, J. C. Rodríguez-Sanjuán, R. A. Domínguez Díez, A. García-Barón Pórtoles, \\ M. S. Trugeda Carrera, F. de la Torre Carrasco and M. Gómez-Fleitas
}

Department of Surgery. University Hospital Marqués de Valdecilla. Santander, Spain

\begin{abstract}
Portal and mesenteric vein thrombosis is a very uncommon complication of laparoscopic surgery, especially after anti-reflux procedures. We report the case of a twenty-year-old man with a history of alcohol and cocaine consumption. A Nissen fundoplication was performed. The patient received a single 20-mg dose of enoxaparin (Clexane ${ }^{\circledR}$, Aventis Pharma, Spain) two hours before surgery for antithrombotic prophylaxis. On the seventh postoperative day the patient had a portal and mesenteric venous thrombosis, which was confirmed at laparotomy, with both extensive small-intestine necrosis and partial colon necrosis. Despite anticoagulant therapy, the patient died 24 hours later. Surgical findings were confirmed at necropsy.

Portal and mesenteric venous thrombosis is an uncommon but severe and even fatal complication after laparoscopic anti-reflux surgery. When other pro-thrombotic, predisposing conditions such as laparoscopic surgery and cocaine consumption are present, the usual prophylactic doses of low molecular weight heparin might not be sufficient to protect against this life-threatening complication.
\end{abstract}

Key words: Portal thrombosis. Mesenteric thrombosis. Nissen fundoplication. Cocaine. Laparoscopy.

García Díaz RA, Rodríguez-Sanjuán JC, Domínguez Díez RA, García-Barón Pórtoles A, Trugeda Carrera MS, de la Torre Carrasco F, Gómez-Fleitas M. Fatal portal thrombosis after laparoscopic Nissen fundoplication. Rev Esp Enferm Dig 2005; 97: 666-669.

Recibido: 14-03-05.

Aceptado: 16-03-05.

Correspondencia: Rosa Ana García Díaz. C/ Rosa, 16, chalet nº 1. 39010 Santander. Cantabria.

\section{INTRODUCTION}

Portal and mesenteric vein is a very uncommon complication of laparoscopic surgery. We report the fourth case to our knowledge after laparoscopic Nissen fundoplication.

\section{CASE REPORT}

We report the case of a twenty-year-old man having a severe gastroesophageal reflux disease, uncontrolled with omeprazol $60 \mathrm{mg}$ daily, and with a history of alcohol and cocaine consumption. Upper endoscopy revealed severe esophagitis and an image suggesting Barrett's esophagus. A severe gastro-esophageal reflux was shown on $\mathrm{pH}$ monitoring with a DeMeester score of 193 (normal < 14.72). Lower esophageal sphincter pressure was $3 \mathrm{mmHg}$ (normal range, $8-25 \mathrm{mmHg}$ ). An antireflux procedure was indicated. Antithrombotic prophylaxis was performed with $20 \mathrm{mg}$ of enoxaparin (Clexane ${ }^{\circledast}$, Aventis Pharma, Spain), one dose two hours before surgery and one dose for 3 additional days. A pneumoperitoneum was carried out with $\mathrm{CO}_{2}$ at a controlled maximum pressure of $14 \mathrm{mmHg}$. A short floppy Nissen fundoplication was done with non-absorbable material after adapting the diaphragmatic crura behind the esophagus. Surgery time was 105 minutes, with no incidents. The first 72-hour of postoperative period was uneventful, and the patient was discharged asymptomatic.

On the seventh postoperative day the patient ingested excessive amounts of alcohol and food, and suddenly developed a clinical picture consisting of severe back pain, hyperpyrexia, abdominal swelling and hemodynamic instability. Laboratory tests showed increased leukocyte count with $12 \%$ immature cells, metabolic acidosis $(\mathrm{pH}$ 7.27), and prolonged prothrombin time. CT findings were small-intestine dilatation, ascites, liver edema, and suspi- 
cion of both mesenteric and portal thrombosis. The patient was operated on with the following findings: extensive small-intestine ischemia and partial colon ischemia, liver edema, portal thrombosis, and superior mesenteric artery spasm with no clot inside, as shown by means of a Fogarti's balloon exploration. Intraoperative Doppler ultrasounds showed total thrombosis and air in the portal vein, as well as patent mesenteric and hepatic arteries. No surgical manoeuvre was performed on the vessels. No data suggesting hypercoagulability was found. Urine cocaine detection was not possible, since no sample from the first hours after admission was available. Despite anticoagulation therapy, the patient exhibited a progressive deterioration and died 24 hours after revision surgery. Necropsy showed extensive thrombosis of both the portal and inferior mesenteric veins with hemorrhagic infarction of the whole small intestine and right colon, acute mucosal ulcers and blood in the intestinal lumen. Liver hemorrhagic infarction, left ventricular hypertrophy and dilatation, and lung focal microcalcifications (a typical finding in chronic cocaine consumers) were also found. Hypovolemic, hemorrhagic shock due to portal and mesenteric thrombosis was established as the cause of death.

\section{DISCUSSION}

Portal and mesenteric thrombosis after laparoscopic surgery is very uncommon. We report the fourth case ever published in the literature to our knowledge, and the first with a fatal outcome. Portal thrombosis has been reported after laparoscopic surgery of the colon, spleen and gallbladder, but very infrequently after Nissen fundoplication, all having a favorable outcome (1-3). Two cases also showed additional predisposing conditions to thrombosis: deep vein thrombosis in a leg (1) and tobacco use (2).

Symptom onset happened after abundant food ingestion in one case (2) and after food and gassy drink ingestion in our case. The onset of the portal thrombosis clinical picture may vary from insidious, consisting of mild pain with scanty physical findings, to one of sudden onset with fever, tachycardia and hypotension, later evolving to a shock, as was the case with our patient. Positive intraabdominal pressure due to pneumoperitoneum may predispose to portal vein occlusion. Jakimowicz et al. showed that portal blood flow decreased to $53 \%$ with a pressure of $14 \mathrm{mmHg}$ (4). High pressure causes collapse and, therefore, blood flow resistance increases (2). Also, hypercapnia due to $\mathrm{CO}_{2}$ absorption brings about a decrease in mesenteric blood flow as a result of vasoconstriction. The effect of all these factors increases with surgery time and is aggravated with the patient's position on the surgery table (4). However, the clinical incidence of portal thrombosis is minimal. We thus believe that there may be another factor playing a central role in its pathogenesis. Technical mistakes were excluded after revising surgery tape records. Could this complication be related to cocaine consumption? The relationship between cocaine consumption and thrombotic and cardiovascular events is well documented, including digestive complications: arterial mesenteric ischemia, mesenteric vein thrombosis, hepatocellular necrosis, peptic ulcer with perforation or ischemic colitis (5). These may be associated with both acute and chronic consumption. Cocaine provokes severe splanchnic vasoconstriction due to its sympathetic action, thus blocking the reuptake and removal of cathecolamines. It also promotes platelet aggregation and erythrocitosis, and therefore predisposes to arteriolar obstruction. As a result, both mucosal ulcers and submucosal hemorrhage appear in the short term, and pseudomembranes in the long term. Cocaine also has a direct toxic effect on the gastric mucosa and delays gastric emptying due to its anticholinergic effect and action on medullary centers (5).

The concurrency of all these factors -laparoscopic surgery, chronic cocaine consumption, abundant gassy drink intake in the immediate postoperative period- increased the risk leading to massive portal thrombosis, which was not prevented by the low molecular weight heparin regimen administered.

Would the fatal outcome have been prevented with a longer heparin regimen, with higher doses? Would a different regimen have diminished the severity of the clinical picture? This is not known and remains speculative.

It is therefore useful to preoperatively find any risk-increasing factors in laparoscopic surgery: hypercoagulability disorders, thrombotic and embolic antecedents, cocaine consumption, and in general any cardiovascular risk factor. Then, a cost-effective prophylactic heparin regimen must be defined.

\section{REFERENCES}

1. Kemppainem E, Kokkola A, Siren J, Kiviluoto T. Superior mesenteric and portal vein trombosis following laparoscopic fundoplication. Dig Surg 2000; 17: 279-81.

2. Davies M, Satyadas T, Akle CA. Spontaneus resolution of a superior mesenteric vein thrombosis after laparoscopic Nissen fundoplication. Ann R Coll Surg Engl 2002; 84: 177-80.

3. Steele SR, Martin MJ, Garafalo T, Ko Tak-ming, Place RJ. Superior mesenteric Vein Thrombosis Following Laparoscopic Nissen Fundoplication. JSLS 2003; 7: 159-63.

4. Jakimovicz J, Stultiens G, Smulders F. Laparoscopic insufflation of the abdomen reduces portal venous flow. Surg Endosc 1998; 12: 129-32.

5. Boghdadi MS, Henning RJ. Cocaine: pathophysiology and clinical toxicology. Heart Lung 1997: 26466-83. 